\title{
Sepsis score performance in women at high and low risk of intraamniotic infection: a retrospective case-control study
}

Hen Y Sela ( $\square$ hysela@szmc.org.il )

Shaare Zedek Medical Center https://orcid.org/0000-0002-9859-469X

Vered Seri

Shaare Zedek Medical Center

Frederic S. Zimmerman

Shaare Zedek Medical Center https://orcid.org/0000-0001-9698-9965

Philip D. Levin

Shaare Zedek Medical Center

Arnon Smueloff

Shaare Zedek Medical Center

Sharon Einav

Shaare Zedek Medical Center

Research article

Keywords: Chorioamnionitis, intraamniotic infection, SIRS, qSOFA, sepsis, early identification

Posted Date: September 1st, 2020

DOl: https://doi.org/10.21203/rs.3.rs-61687/v1

License: (9) (i) This work is licensed under a Creative Commons Attribution 4.0 International License. Read Full License 


\section{Abstract}

Background: Early infection identification may improve outcomes in obstetric patients. However, obstetric vital signs and laboratory values differ from the non-pregnant, possibly limiting current sepsis score use. Thus, we evaluated sepsis score use in peripartum infection.

Methods: This case-control study evaluates sepsis criteria fulfilment in preterm premature rupture of membranes (PPROM) - at high infection risk- versus in elective caesarean delivery (CD) - at low risk. The study was perfoemd at the departement of obstetrics \& gynecologt at Shaare Zedek Medical Center, a 1000-bed university-affiliated acute care hospital. We inlcuded women with PPROM undergoing CD $(n=453)$ at gestational weeks $24-36$ versus those undergoing elective term CD $(n=2004)$. The primary and secondary outcome measures were SIRS and qSOFA criteria fullfilment and availability of the score components and clinical intraamniotic infection and positive cultures rates.

Results: At admission $14.8 \%$ of the study group and $4.6 \%$ of control met SIRS criteria $(p=0.001)$, as did $12.5 \%$ and $5.5 \%$ on post-operation day (POD) 1 ( $p=0.001$ ), with no significant differences on POD 0 or 2 . In the study group more cultures $(29.8 \%$ versus $1.9 \%$ - cervix; $27.4 \%$ versus $1.1 \%$ - placenta; $7.5 \%$ versus $1.7 \%$ - blood; $p=0.001$ - all differences) and more positive cultures ( $5.5 \%$ versus $3.0 \%$ - urine $-p=0.008$; $4.2 \%$ versus $0.2 \%$ - cervix $-p=0.001 ; 7.3 \%$ versus $0.0 \%$ - placenta $-p=0.001 ; 0.9 \%$ versus $0.1 \%-$ blood - $p=0.008$ ) were obtained. $10.6 \%$ of the study group and $0.4 \%$ of control met criteria for intraamniotic infection $(p=0.001)$.

Conclusions: Though significant difference was noted in SIRS criteria fulfilment in the study group versus control, there was considerable between-group overlap, questioning utility of SIRS in intraamniotic infection diagnosis.

\section{Background}

Early warning scores are associated with improved sepsis outcomes and are important in early infection assessment [1,2]. Pregnant women are at increased risk of infection, the third most common cause of maternal death [3]. However, vital signs and laboratory values of pregnant women differ from the nonpregnant, creating uncertainty regarding the value of current sepsis scores in pregnancy.

Generic scoring systems may facilitate early recognition of critical illness, including sepsis, in obstetric patients $[4,5]$. However, these tools are non-specific. Systemic inflammatory response syndrome (SIRS) criteria had been recommended for early sepsis identification $[2,6,7]$. In 2016, quick sequential organ failure assessment (qSOFA) was recommended to replace SIRS. qSOFA reflects organ failure severity rather than immune response to infection and was proposed as a more evidence-based and clinically practical means of recognizing severe infection [8].

Intraamniotic infection (IAI) is an important infection in the obstetric population, resulting in maternal morbidity and mortality and fetal loss. Early antibiotic treatment may improve outcomes, making early 
identification vital $[5,9]$. Preterm premature rupture of membranes (PPROM) is associated with increased risk of IAI $[10,11]$.

Thus, the current study investigates use of sepsis criteria in IAI identification. The primary objective was to evaluate SIRS and qSOFA criteria fulfilment among peripartum women with PPROM versus those at low risk. Secondary objectives were to evaluate the actual availability of score components and the rate of documented IAI and positive cultures in the two groups.

\section{Methods}

Following Shaare Zedek Medical Center (SZMC) IRB approval (SZMC-0001-16) and given that the study was based on patient records, informed consent was waived. A retrospective case-control study was conducted on women who underwent caesarean delivery (CD) between August 2005 and December 2015 in SZMC, Jerusalem, Israel.

\section{Clinical setting}

The SZMC is a 1000-bed university-affiliated acute care hospital with a Division of Obstetrics that includes a high risk pregnancy unit, 2 delivery suites with 16 delivery rooms attached dedicated two obstetric operating rooms and 4 maternity wards. There were approximately 14,000 annual delivery admissions during the study period.

Inclusion/exclusion criteria: The study group consisted of women admitted with PPROM at gestational weeks (GW) 24-36 undergoing CD within 7 days of admission and prior to GW37. Previous studies have demonstrated a $10 \%$ risk of IAI in this population [10]. Control consisted of women undergoing CD at GW37-40, with no rupture of membranes or trial of labour. Intraamniotic infection risk in this population is considered approximately $1.7 \%$ [12], though little quality data exists. Vaginal delivery, rupture of membranes prior to GW24, rupture of membranes or trial of labour after GW37, delivery within 24 hours of PPROM and delivery $>7$ days after admission were exclusion criteria. Case identification: Cases were identified via structured queries to the obstetric electronic medical record (EMR - NeSS Technologies, Israel). For the study group, the query identified women admitted during the study period who (1) had undergone CD prior to GW37 within one week of admission and (2) had an admission or discharge rupture of membranes diagnosis. If discrepancy was noted between admission and discharge diagnoses, the medical record was reviewed; if rupture of membranes was not recorded on admission or throughout hospitalization, the case was excluded. Data missing from the EMR were completed from hard copy files.

For control, the query identified women undergoing CD at GW37-40 with no trial of labour. Due to a large quantity of eligible subjects, those without full EMR data (as described below) were excluded, without hard copy review.

\section{Variables}


Primary outcomes were (1) SIRS and qSOFA criteria fulfilment among the study population and (2) as compared to control. Secondary outcomes included (a) availability of the components of the above scores and (b) rates of clinical intraamniotic infection and positive cultures (urine, cervix, placenta and blood) in the two groups.

Thus, vital signs (heart rate, blood pressure, temperature, respiratory rate, mental status) as documented by nursing staff, complete blood count $(\mathrm{CBC})$, creatinine, bilirubin and culture results were collected at admission and on POD 0,1 and 2 .

For purposes of this study, IAI was defined as hospital discharge with a relevant diagnostic code (ICD-9 658.41) or broad spectrum antibiotic treatment during labor and delivery. Though recent evidence advocates additonal of azithromycin to the usal prophylactic antibiotics in many or all women undergoing CD [14], our institutional protocols at the time of the study, in accordance with the best available evidence [15], dictated only narrow spectrum antibiotics as CD surgical prophylaxis and Broad spectrum antibiotics usually Amoxicillin/clavulanate and Gentamycin are used for IAI. Thus, broad spectrum antibiotic use in the obstetric patient in the context of our antibiotic stewardship protocols [16] indicated clinical IAl.

\section{Potential sources of bias}

During data collection it was discovered that temperature was not recorded on POD0 for 88/453 (19.4\%) of the study group and 900/2004 (44.9\%) of control. Upon review, these omissions were noted in women undergoing an evening operation with maternity ward arrival close to midnight. Since these cases comprised a considerable percentage of our study population and time of omission was consistent, concerns arose regarding potential documentation bias. Therefore, women with missing data were compared to those without missing data (Appendix Table 1). The proportion of documentation omissions with regards to blood pressure, heart rate and temperature excluding Post operative day (POD) 0 was negligible and random, therefore women lacking data regarding any of these were excluded from the study (Appendix Table 2). An additional issue was a complete lack of respiratory and mental status documentation patients in either group, precluding calculation of qSOFA. 
Table 1

Demographic, obstetric and medical characteristics of study group versus control group. PPROM: preterm premature rupture of membranes; Cl: confidence interval

\begin{tabular}{|llll|}
\hline Characteristic & PPROM & Control $\mathbf{n = 2 0 0 4}(\%)$ & $\mathbf{p}$ \\
& $\mathbf{n = 4 5 3}(\%)$ & \\
\hline Maternal age (mean $\pm \mathrm{Cl})$ & $31.5 \pm 6.7$ & $33.8 \pm 5.3$ & 0.001 \\
\hline$>35$ year & $127(28.0)$ & $750(37.4)$ & 0.001 \\
\hline Member of minority & $70(15.5)$ & $220(11.0)$ & 0.001 \\
\hline Completed secondary education & $411(90.7)$ & $1934(96.5)$ & 0.005 \\
\hline Gestation number & $3.2 \pm 2.5$ & $3.9 \pm 2.6$ & 0.001 \\
\hline Previous caesareans $($ mean $\pm \mathrm{Cl})$ & $0.4 \pm 0.8$ & $1.1 \pm 1.2$ & 0.001 \\
\hline Any previous caesarean & $121(26.7)$ & $1077(59.9)$ & 0.001 \\
\hline Previous abortion $($ mean $\pm \mathrm{Cl})$ & $0.7 \pm 1.3$ & $0.7 \pm 1.1$ & 0.531 \\
\hline In vitro fertilization & $126(27.8)$ & $319(15.9)$ & 0.001 \\
\hline Twin pregnancy & $160(35.3)$ & $200(10.0)$ & 0.001 \\
\hline Gestational diabetes & $46(10.2)$ & $269(13.4)$ & 0.060 \\
\hline Hypertension & $18(4.0)$ & $69(3.4)$ & 0.581 \\
\hline Hypothyroidism & $29(6.4)$ & $117(5.8)$ & 0.647 \\
\hline
\end{tabular}


Table 2

Vital signs and white cell counts of study group versus control group. PPROM: preterm premature rupture of membranes; POD: post-operation day

\begin{tabular}{|c|c|c|c|c|c|c|}
\hline Characteristic & Day & PPROM & $\mathbf{n}$ & Control & $\mathrm{n}$ & p \\
\hline \multirow[t]{4}{*}{ Temperature } & Admission & $36.73 \pm 0.4$ & 453 & $36.68 \pm 0.3$ & 2004 & 0.005 \\
\hline & PODO & $36.72 \pm 0.5$ & 365 & $36.46 \pm 0.5$ & 1104 & 0.001 \\
\hline & POD1 & $36.82 \pm 0.5$ & 453 & $36.81 \pm 0.4$ & 2004 & 0.367 \\
\hline & POD2 & $36.69 \pm 0.4$ & 453 & $36.65 \pm 0.4$ & 2004 & 0.147 \\
\hline \multirow[t]{4}{*}{ Pulse } & Admission & $92.7 \pm 14.4$ & 453 & $88.2 \pm 11.4$ & 2004 & 0.001 \\
\hline & PODO & $84.8 \pm 12.5$ & 453 & $83.2 \pm 10.8$ & 2004 & 0.06 \\
\hline & POD1 & $90.6 \pm 11.5$ & 453 & $88.8 \pm 9.2$ & 2004 & 0.029 \\
\hline & POD2 & $88.9 \pm 11$ & 453 & $87.8 \pm 9.6$ & 2004 & 0.34 \\
\hline \multirow[t]{4}{*}{ Mean arterial pressure } & Admission & $90.3 \pm 10.9$ & 453 & $85.6 \pm 9.3$ & 2004 & 0.001 \\
\hline & PODO & $76.8 \pm 10.5$ & 453 & $74.3 \pm 9.5$ & 2004 & 0.001 \\
\hline & POD1 & $73.6 \pm 9.5$ & 453 & $70.8 \pm 8.2$ & 2004 & 0.001 \\
\hline & POD2 & $78.2 \pm 10$ & 453 & $76.8 \pm 9.1$ & 2004 & 0.008 \\
\hline \multirow[t]{4}{*}{ Leukocyte count $\left({ }^{\star} 10^{3}\right)$} & Admission & $10.3 \pm 9.2$ & 452 & $9.2 \pm 2.2$ & 1997 & 0.001 \\
\hline & PODO & $13.4 \pm 11.7$ & 310 & $11.7 \pm 3.3$ & 1617 & 0.001 \\
\hline & POD1 & $12.2 \pm 11.4$ & 187 & $11.4 \pm 3$ & 587 & 0.007 \\
\hline & POD2 & $11.4 \pm 9.7$ & 56 & $9.7 \pm 2.6$ & 138 & 0.005 \\
\hline
\end{tabular}

\section{Data collection}

SZMC has separate EMRs for obstetric and all other patient data. Cases were identified and vital signs collected from the obstetric EMR and other data from the hospital-wide EMR. As noted above, data unavailable in the EMR was obtained from hard copy files.

For all women, initial data included variables at time of admission. For women not undergoing $C D$ at admission, data were collected from the high risk pregnancy unit. Women who remained in the delivery suite more than 24 hours before $C D$ had data collected there. All data collected after CD were from maternity ward notes.

Sample size considerations: The study was designed as a 1:4 case-control study. Based on previous studies, we hypothesized a $10 \%$ IAI rate in the study group[17], with an infection rate of at least $1.7 \%$ and 
no more than 5\% in the control [12]. Thus, 437 cases in the study group and 1748 in the control were needed to reject (with a power of 0.95) the null hypothesis of an identical rate of IAI in the study and control groups. Upon initial database review, we identified approximately 600 women with a diagnosis of PPROM. We assumed that about $30 \%$ would be eliminated on review. Thus 420 would remain in the study group, of whom approximately $42(10 \%)$ would be found to have an IAI. No more than 2000 subjects were needed in the control group, of whom no more than 100 (5\%) would be found to have an IAl. The probability of a type I error associated with this test of the null hypothesis was calculated as 0.05 .

\section{Quantitative variables}

The lowest systolic blood pressure and highest pulse and fever recorded each day were used for analysis. Glasgow Coma Scale was to be described as the total sum rather than its components. Laboratory data, including leukocyte and platelet count, creatinine and bilirubin, were collected and analysed as presented by the hospital laboratory.

Data management and statistical analysis. Data were downloaded from the EMR to a Microsoft Excel (Version 2010) database and then transferred to SPSS (Version 22.0. Armonk, NY: IBM Corp.), which was used for analysis. Data from hard copy files were manually added to the database.

In the first step, descriptive statistics (i.e. numbers, proportion and means) were used to describe the study population as a whole, as well as study and control group characteristics (Table 1).

In the second step, comparisons between women with and without missing data were performed and in the third step, comparisons between study and control groups were performed. Comparison between proportions was performed using the $\chi^{2}$-score (e.g. for demographic and clinical features) or the Fisher's exact test (e.g. for the rate of positive cultures). To compare continuous variables the Student's t-test (e.g. for maternal age) or the Mann-Whitney-Wilcoxon test (e.g. for vital signs and obstetric characteristics such as number of gestations and previous $\mathrm{CDs}$ ) were used. In all tests, two-tailed p-values were taken and a $p$-value $<0.05$ was considered significant.

Finally, we performed sensitivity analyses comparing positive urinary culture rates between groups.

\section{Results}

Of 142,372 study period deliveries, 7,499 deliveries occurred during GW24-36. Among these, 621 women with a presumptive PPROM diagnosis underwent CD. After chart review, 168 did not fulfil study group criteria. Likewise, 107,401 study period deliveries took place during GW37-40. Among these, 3159 women underwent $C D$ without trial of labour. After chart review, 1155 did not fulfill control group criteria. Thus, the study group included 453 subjects and the control, 2004 (Fig. 1).

\section{Reporting bias}


Comparison of cases with missing data to those without is presented in Appendix Table 1. In the control, patients with missing data were slightly younger, had fewer previous pregnancies and CDs and a higher incidence of hypertension. No significant differences were noted in the study group.

\section{Study population description}

Study population age was $33.6 \pm 5.6$ years, with $3.8 \pm 2.6$ previous deliveries and $1.1 \pm 0.9$ previous CDs. Women in the study group were younger, more likely to be a minority, less likely to have completed secondary education and had fewer deliveries and CDs (Table 1).

\section{Sepsis criteria}

Documentation of vital signs and CBC was high (>98\%) in both groups, with no significant betweengroup differences. The study group had a higher temperature at admission and on POD0, with higher mean arterial pressures and leukocyte counts throughout admission. Although statistically significant, these differences were clinically meaningless. The study group had higher heart rates throughout admission, with statistical significance varying by day (Table 2 ).

$14.8 \%(67 / 453)$ of the study group and $4.6 \%(92 / 2004)$ of control fulfilled SIRS criteria at admission ( $p=$ $0.001)$, with $12.5 \%(57 / 453)$ versus $5.5 \%(110 / 2004)$ SIRS criteria fulfilment on POD1 ( $p=0.001)$ and no significant differences on other hospitalization days (Fig. 2). Medical record data did not suffice for calculating qSOFA scores.

\section{Clinical IAI}

$48 / 453(10.6 \%)$ of the study group and $8 / 2004(0.4 \%)$ of control met clinical criteria for intraamniotic infection $(p=0.001)$.

\section{Culture comparisons}

Urine cultures were obtained from $91.4 \%$ (414/453) of the study group and $100.0 \%(2004 / 2004)$ of control $(p=0.001)$. It should be noted that our institutional protocol mandates urine culture obtainment from all patients undergoing CD. Obtainment of cervical, placental and blood cultures was considerably lower in both groups. Significantly more cultures were obtained from the study group versus control. Significantly more positive cultures of all types were noted in the study group versus control - see Table 3. 
Table 3

Microbiological cultures - study group versus control group. PPROM: preterm premature rupture of membranes

\begin{tabular}{|lllllll|}
\hline $\begin{array}{l}\text { Body } \\
\text { site }\end{array}$ & \multicolumn{2}{l}{ Cultures obtained } & \multicolumn{4}{l|}{ Positive cultures } \\
\cline { 2 - 6 } & $\begin{array}{l}\text { PPROM }(\mathbf{n}= \\
\text { 453) }\end{array}$ & $\begin{array}{l}\text { Control }(\mathbf{n}= \\
\text { 2004) }\end{array}$ & $\mathbf{p}$ & $\begin{array}{l}\text { PPROM }(\mathbf{n}= \\
\mathbf{4 5 3})\end{array}$ & $\begin{array}{l}\text { Control }(\mathbf{n}= \\
\text { 2004) }\end{array}$ & $\mathbf{p}$ \\
\hline Urine & $414(91.4)$ & $2004(100.0)$ & 0.001 & $25(5.5)$ & $60(3.0)$ & 0.008 \\
\hline Cervix & $135(29.8)$ & $38(1.9)$ & 0.001 & $19(4.2)$ & $4(0.2)$ & 0.001 \\
\hline placenta & $124(27.4)$ & $22(1.1)$ & 0.001 & $33(7.3)$ & $1(0.0)$ & 0.001 \\
\hline blood & $34(7.5)$ & $34(1.7)$ & 0.001 & $4(0.9)$ & $3(0.1)$ & 0.008 \\
\hline
\end{tabular}

\section{Sensitivity analysis - urinary cultures}

A sensitivity analysis was performed for urinary cultures. Analysis assuming positive missing cultures resulted in increased difference between the study group and control; whereas analysis assuming negative missing cultures resulted in decreased between-group difference but remained statistically significant (Appendix Table 3).

\section{Discussion}

Sepsis, especially due to IAI, increases in PPROM and early diagnosis of severe infection is particularly important in this population [18]. This retrospective study of 2457 women examined the proportion of PPROM patients meeting SIRS and qSOFA criteria versus a low-risk population. Significantly more high risk women versus low risk met SIRS criteria, especially on admission. However, there were women in both groups that did not meet criteria despite differences in risk and eventual culture positivity. In other words, peripartum women with high infection risk may not necessarily fulfil SIRS criteria while those fulfilling criteria may not be at risk. These findings are similar to a previous retrospective study investigating intraamniotic infection, which failed to show an association between SIRS criteria and intensive care unit transfer [17]. Unlike the previous study, we evaluated SIRS criteria for the majority of patients (Fig. 1), comparison was done to control and analysis of sepsis criteria was performed at multiple points. Our study supports previous studies suggesting that physiological changes of pregnancy complicate identification of infection $[19,20]$.

Though there are accepted normal ranges in pregnancy for components of the SIRS criteria, these are based on expert consensus only and differ significantly from normal ranges of the non-pregnant population $[9,20]$. This study demonstrated a statistically significant between-group difference in SIRS criteria, maintained at several time points throughout admission. Nevertheless, a substantial percentage of the study group did not meet SIRS criteria whereas a substantial percentage of the control did. As such, these criteria do not seem useful in identifying women at risk. 
An important secondary finding is the extent of missing documentation. As in other studies [21, 22], relevant maternal parameters (particularly respiratory rate and mental status) were not documented. This may be related to long-standing midwifery traditions [22] and the tendency, as described in other areas [23], to treat the pregnancy rather than the pregnant woman. As such, though qSOFA is recommended for sepsis identification precisely because its components are used to monitor general ward patients, this assumption does not appear to be true in obstetric populations. The question arises whether these measurements should be incorporated into obstetric work routine.

Despite between-group similarity, more blood, placental and vaginal cultures were taken from the study group. In our institution, these are obtained only upon clinical suspicion of infection. These suspicions were substantiated by an associated higher positive culture rate. In absence of clear-cut differences, the question remains as to what led clinicians to suspect infection. If this is quantifiable, perhaps clearer indicators for prompt diagnosis can be established.

In this study, as in previous studies [17], $10 \%$ of those with PPROMM developed intrauterine infection and rates of positive urinary, vaginal and placental cultures were similar to those described in previous studies $[10,24]$. Additionally, our control group positive urinary culture rates similar to previous studies [25-27]. These similarities increase the generalizability of our findings.

This study has several limitations. As a retrospective analysis, its conclusions should be approached cautiously. This is reinforced by demographic differences between the study and control groups (Table 1). A further limitation is the lack of full documentation, necessitating elimination of a substantial percentage of the initial cohort, and making it impossible to evaluate qSOFA. In addition, cultures obtainment rate was lower than expected, with more positive cultures in the study group versus control. Furthermore, in order to simplify comparisons, this study was conducted on patients undergoing CD only, limiting applicability to vaginal delivery. Another consideration is the lack of very severe illness in the study population, with only 7 positive blood cultures and no intensive care unit admissions.

In conclusion, this study found that though there is a statistically significant difference in the rate of SIRS in patients with PPROM undergoing CD versus those undergoing elective $C D$, there is still considerable overlap between these two groups, calling into question the utility of SIRS in prompt diagnosis of IAI in peripartum women. This study also emphasizes the need for better maternal monitoring in the peripartum period and more widespread use of cultures in identifying intraamniotic infection in at-risk populations.

\section{List Of Abbreviations}

SIRS - Systemic inflammatory response syndrome

qSOFA - quick sequential organ failure assessment

IAI - Intraamniotic infection

PPROM - Preterm premature rupture of membranes 
SZMC - Shaare Zedek Medical Center

CD - caesarean delivery

GW - gestational weeks

EMR - electronic medical record

POD - Post operative day

\section{Declarations}

Ethics approval - This study was approved by Shaare Zedek Medical Center Institutional Review Board. As the study was based on patient records, informed consent was waived.

Consent for publication (include appropriate statements) - Not applicable.

Availability of data and material (data transparency) - All data is avilable upon request and masking of personal and identifing data form the corresponding author.

Competing interests - The authors report no conflict of interest.

Funding - This research received no specific grant from any funding agency in the public, commercial or not-for-profit sectors.

Authors' contributions: HYS: Protocol development, Data collection, Data analysis, Manuscript writing; VS: Protocol development, Data collection, Data analysis, Manuscript writing; FSZ: Data analysis, Manuscript writing; PDL: Protocol development; AS: Protocol development, Data collection; SE: Protocol development, Data collection, Data analysis, Manuscript writing. All authors have read and approved the manuscript.

Acknowledgements - Not Applicable

\section{References}

1. Levy MM, Evans LE, Rhodes A (2018) The Surviving Sepsis Campaign Bundle: 2018 update. Intensive Care Med 44:925-928. https://doi.org/10.1007/s00134-018-5085-0

2. Levy MM, Dellinger RP, Townsend SR, et al (2010) The surviving sepsis campaign: Results of an international guideline-based performance improvement program targeting severe sepsis. Intensive Care Med 36:222-231. https://doi.org/10.1007/s00134-009-1738-3

3. Creanga AA, Syverson C, Seed K, Callaghan WM (2017) Pregnancy-related mortality in the United States, 2011-2013. Obstet Gynecol 130:366-373. https://doi.org/10.1097/AOG.0000000000002114 
4. Singh S, McGlennan A, England A, Simons R (2012) A validation study of the CEMACH recommended modified early obstetric warning system (MEOWS). Anaesthesia 67:12-18. https://doi.org/10.1111/j.1365-2044.2011.06896.x

5. Isaacs RA, Wee MYK, Bick DE, et al (2014) A national survey of obstetric early warning systems in the United Kingdom: five years on. Anaesthesia 69:687-692. https://doi.org/10.1111/anae.12708

6. Balk RA, Bone RC (1989) The septic syndrome. Definition and clinical implications. Crit Care Clin 5:1-8. https://doi.org/10.1097/00002820-199114040-00002

7. Bone RC, Balk RA, Cerra FB, et al (1992) Definitions for sepsis and organ failure and guidelines for the use of innovative therapies in sepsis. Chest 101:1644-1655.

https://doi.org/10.1378/chest.101.6.1644

8. Singer M, Deutschman CS, Seymour C, et al (2016) The third international consensus definitions for sepsis and septic shock (Sepsis-3). JAMA 315:801-810. https://doi.org/10.1001/jama.2016.0287

9. Higgins RD, Saade G, Polin RA, et al (2016) Evaluation and management of women and newborns with a maternal diagnosis of chorioamnionitis. Obstet Gynecol 127:426-436. https://doi.org/10.1097/AOG.0000000000001246

10. Arora P, Bagga R, Kalra J, et al (2015) Mean gestation at delivery and histological chorioamnionitis correlates with early-onset neonatal sepsis following expectant management in pPROM. J Obstet Gynaecol (Lahore) 35:235-240. https://doi.org/10.3109/01443615.2014.958143

11. (2018) ACOG practice bulletin No. 188: prelabor rupture of membranes (PROM). Obs Gynecol 131:e1$-14$

12. Kamath BD, Todd JK, Glazner JE, et al (2009) Neonatal outcomes after elective cesarean delivery. Obstet Gynecol 113:1231-1238. https://doi.org/10.1097/AOG.0b013e3181a66d57

13. Ganer H, Levy A, Ohel I, Sheiner E (2009) Pregnancy outcome in women with an intrauterine contraceptive device. Am J Obstet Gynecol 201:381.e1-381.e5. https://doi.org/10.1016/j.ajog.2009.06.031

14. Tita ATN, Szychowski JM, Boggess K, et al (2016) Adjunctive Azithromycin Prophylaxis for Cesarean Delivery. N Engl J Med 375:1231-1241. https://doi.org/10.1056/NEJMoa1602044

15. Smaill FM, Grivell RM (2014) Antibiotic prophylaxis versus no prophylaxis for preventing infection after cesarean section. Cochrane Database Syst Rev.

https://doi.org/10.1002/14651858.CD007482.pub3

16. Yinnon AM (2001) Whither Infectious Diseases Consultations? Analysis of 14,005 Consultations from a 5-Year Period. Clin Infect Dis 33:1661-1667. https://doi.org/10.1086/323760

17. Lappen JR, Keene M, Lore M, Grobman WA (2010) Existing models fail to predict sepsis in an obstetric population with intrauterine infection. Am J Obstet Gynecol 203:573e1-e5. https://doi.org/http://dx.doi.org/10.1016/j.ajog.2010.07.040

18. Bauer ME, Bateman BT, Bauer ST, et al (2013) Maternal sepsis mortality and morbidity during hospitalization for delivery. Anesth Analg 117:944-950.

https://doi.org/10.1213/ANE.0b013e3182a009c3

Page 12/15 
19. Bauer ME, Bauer ST, Rajala B, et al (2014) Maternal physiologic parameters in relationship to systemic inflammatory response syndrome criteria: A systematic review and meta-analysis. Obstet Gynecol 124:535-541. https://doi.org/10.1097/AOG.0000000000000423

20. Gat R, Hadar E, Orbach-Zinger S, et al (2019) Distribution of Extreme Vital Signs and Complete Blood Count Values of Healthy Parturients. Anesth Analg 129:1595-1606. https://doi.org/10.1213/ANE.0000000000003866

21. Bauer ME, Housey M, Bauer ST, et al (2019) Risk Factors, Etiologies, and Screening Tools for Sepsis in Pregnant Women: A Multicenter Case-Control Study. Anesth Analg 129:1613-1620. https://doi.org/10.1213/ANE.0000000000003709

22. Mackintosh N, Watson K, Rance S, Sandall J (2014) Value of a modified early obstetric warning system (MEOWS) in managing maternal complications in the peripartum period: an ethnographic study. BMJ Qual Saf 23:26-34. https://doi.org/10.1136/bmjqs-2012-001781

23. Sela HY, Weiniger CF, Hersch M, et al (2011) The pregnant motor vehicle accident casualty: Adherence to basic workup and admission guidelines. Ann Surg 254:346-352. https://doi.org/10.1097/SLA.0b013e31822638d4

24. Karat $C$, Madhivanan $P$, Krupp K, et al (2006) The clinical and microbiological correlates of premature rupture of membranes. Indian J Med Microbiol 24:283-285. https://doi.org/10.4103/02550857.29388

25. Horowitz E, Yogev Y, Ben-Haroush A, et al (2004) Urine culture at removal of indwelling catheter after cesarean section. Int J Gynecol Obstet 85:276-278. https://doi.org/10.1016/j.ijgo.2003.11.011

26. Atacag T, Yayci E, Guler T, et al (2015) Asymptomatic bacteriuria screened by catheterized samples at pregnancy term in women undergoing cesarean delivery. Clin Exp Obstet Gynecol 42:590-594. https://doi.org/10.12891/ceog1906.2015

27. Nasr AM, ElBigawy AF, Abdelamid AE, et al (2009) Evaluation of the use vs nonuse of urinary catheterization during cesarean delivery: A prospective, multicenter, randomized controlled trial. $J$ Perinatol 29:416-421. https://doi.org/10.1038/jp.2009.4

\section{Figures}




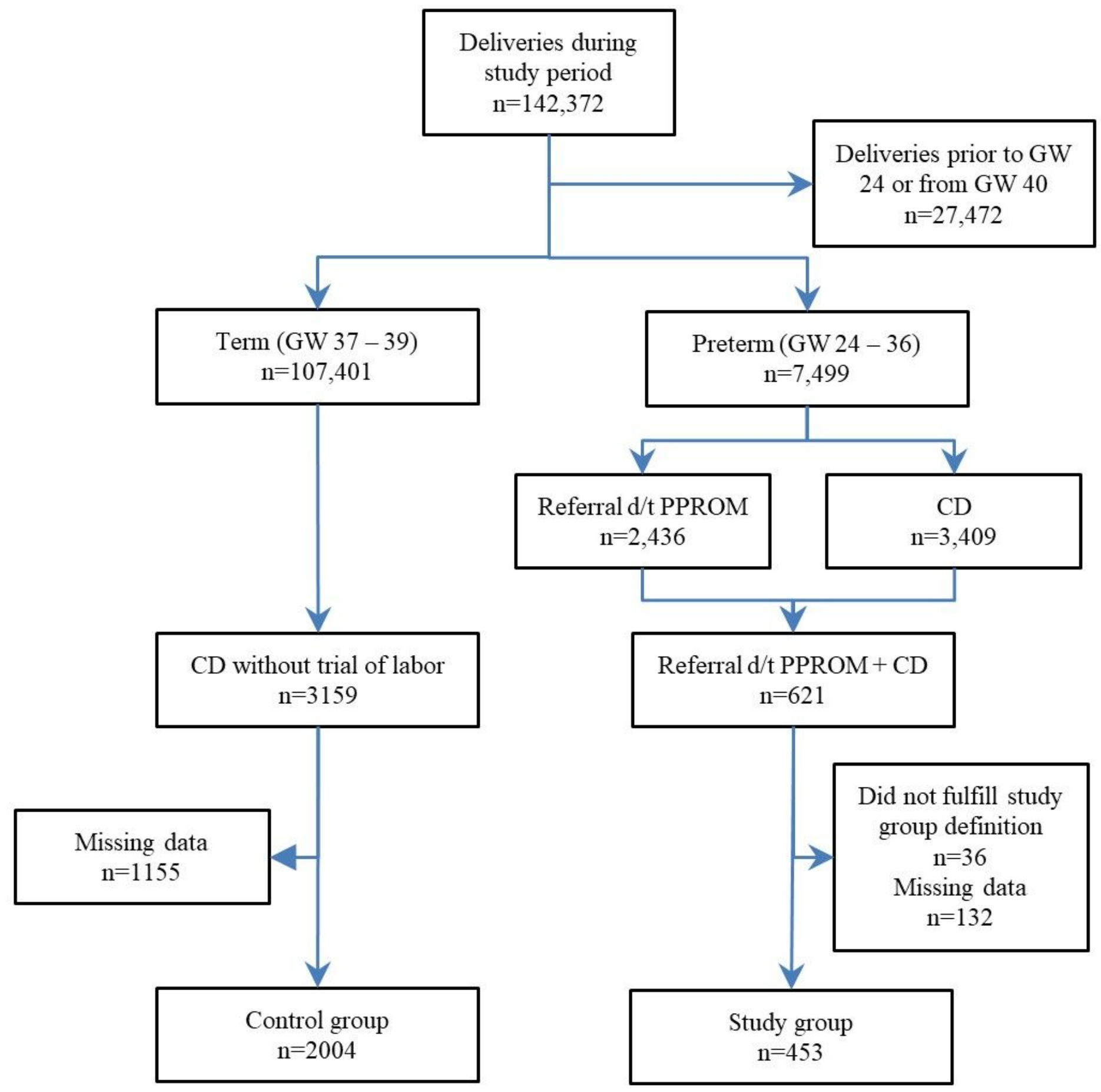

Figure 1

Study inclusion/exclusion process. GW: gestational week; CD: caesarean delivery; PPROM: preterm premature rupture of membranes. 


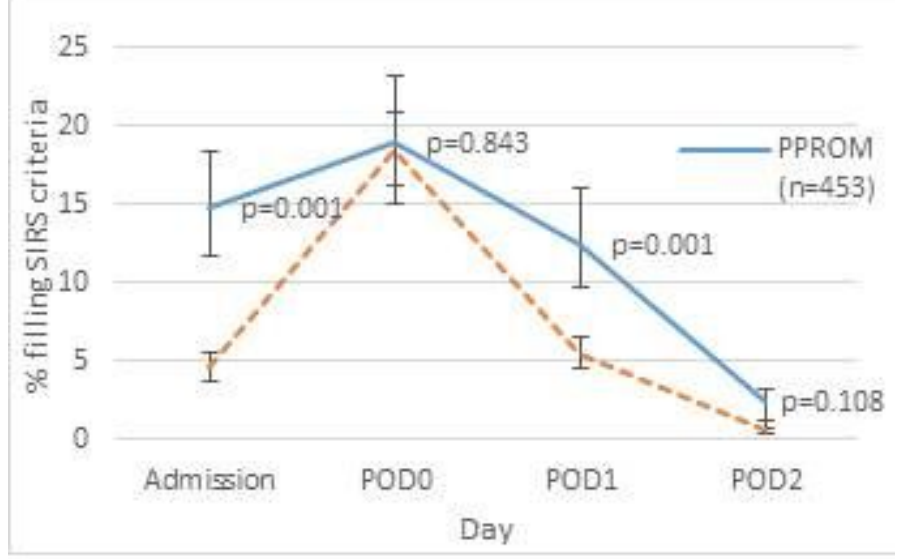

Figure 2

Patients filling systemic inflammatory response syndrome (SIRS) criteria. POD: post-operative day; PPROM: preterm premature rupture of membranes.

\section{Supplementary Files}

This is a list of supplementary files associated with this preprint. Click to download.

- intrauterineinflamationinfectionappendix.pdf 